\title{
Hierarchical price coordination of heat pumps in a building network controlled using model predictive control
}

\author{
Sebastian Gonzato a,b,*, Joseph Chimento ${ }^{a}$, Edward O’Dwyer ${ }^{a}$, Gonzalo Bustos-Turu ${ }^{a}$, \\ Salvador Acha ${ }^{a}$, Nilay Shah ${ }^{\mathrm{a}}$ \\ ${ }^{a}$ Centre for Process Systems Engineering and Department of Chemical Engineering, Imperial College London, United Kingdom \\ ${ }^{\mathrm{b}}$ Energy and Environment, Division of Applied Mechanics and Energy Conversion, Department of Mechanical Engineering, Katholieke Universiteit Leuven, \\ Belgium
}

\section{A R T I C L E I N F O}

\section{Article history:}

Received 6 May 2019

Revised 26 July 2019

Accepted 5 September 2019

Available online $\mathrm{xxx}$

\section{Keywords:}

Model predictive control

Hierarchical control

Heat pump

Heating

Ventilation and air conditioning control

Space heating

Optimal heating strategies

\begin{abstract}
A B S T R A C T
Decarbonisation of the building sector is driving the increased use of heat pumps. As increased electrification of the heating sector leads to stress on the electricity grid, the need for district level coordination of these heat pumps emerges. This paper proposes a novel hierarchical coordination methodology, in which a price coordinator reduces the total instantaneous power demand of a building network below a power supply limit using a price signal. Each building has a model predictive controller (MPC) which maximises thermal comfort and minimises electricity costs. An additional term in the MPC objective function penalises the heat pump power demand quadratically, which when multiplied by a pseudo electricity price allows the price coordinator to reduce the peak power demand of the building network. A 2 building network is studied to analyse the price coordinator algorithm's behaviour and demonstrate how this approach yields a trade off between comfort, energy consumption and peak demand reduction. A 100 building network case study is then presented as a proof of concept, with the price coordinator approach yielding results similar to that of a centralised controller (less than $0.7 \%$ increase in energy consumption per building per year) and a roughly fourfold decrease in computation time.
\end{abstract}

(C) 2019 Elsevier B.V. All rights reserved.

\section{Introduction}

\subsection{Motivation}

Residential and commercial buildings are major energy consumers, accounting for around $20-40 \%$ of global energy consumption [1]. Shaikh et al. [2] estimates that between $5 \%$ and $30 \%$ of this consumption is due to inefficient control strategies for achieving adequate internal comfort. Given the need to decarbonise across society, government policies with the aim of improving energy efficiency in the heating, ventilation and air conditioning (HVAC) systems of buildings are being issued. For example, the 2010 European Energy Performance of Buildings Directive mandates that new private buildings must be almost 'zero-energy' by 2020 [3]. In a policy paper on decarbonising the HVAC sector in the EU by Connolly et al. [4], district heating networks and electrification (e.g. using heat pumps) are identified as key strategies. This electrifica-

\footnotetext{
* Corresponding author at: Energy and Environment, Division of Applied Mechanics and Energy Conversion, Department of Mechanical Engineering, Katholieke Universiteit Leuven, Belgium.

E-mail address: sebastian.gonzato@kuleuven.be (S. Gonzato).
}

tion, along with the energy efficiency gains that can be made using improved control strategies, justifies research into the control and coordination of heat pumps and smart energy systems more generally [5].

\subsection{Economic model predictive control of building HVAC systems}

While control of building HVAC systems has traditionally been done using simple control strategies such as on/off or PID controllers [6, Chapter 4], in the last two decades interest in the use of Model Predictive Control (MPC) for this purpose has increased. MPC uses disturbance predictions (e.g. weather forecasts, estimated occupancies) and estimations of the current state in order to determine the set of system inputs (e.g. heat pump or air conditioning part load) which produce a desired set of predicted system outputs (e.g. zone temperature and humidity). The system inputs are determined by optimising a set of criteria (e.g. minimising costs) while respecting the system (i.e. building) dynamics and input and output constraints (e.g. technology output bounds and thermal comfort bounds respectively) over a receding prediction horizon. The solution of the optimisation problem is the optimal input trajectory, from which the first elements are applied 


\section{Nomenclature \\ Acronyms \\ CMPC Centralised Model Predictive Control or Controller \\ DMPC Distributed Model Predictive Control or Controller \\ EMPC Economic Model Predictive Control or Controller \\ HVAC Heating, Ventilation and Air Conditioning \\ MPC Model Predictive Control or Controller \\ PID Proportional-Integral-Derivative}

Indices and Sets

$b \in \mathcal{B} \quad$ Building index and set

$i \in[0, N-1] \subset \mathbb{Z}$ Time step in MPC prediction horizon index and interval

$j \subset \mathbb{Z} \quad$ Price iteration index

$k \in\left[0, n_{t}\right] \subset \mathbb{Z} \quad$ Simulation time step index and interval

Parameters

$\bar{A}_{b} \quad$ State matrix for discretised state space building

model of building $b[1 / \mathrm{s}]$

$\bar{B}_{b} \quad$ Input matrix for discretised state space building model of building $b[\mathrm{~K} / \mathrm{kJ}]$

$d_{i \mid k} \quad$ External temperature at time step $i$ in the MPC prediction horizon given simulation time step $k$ [K]

$\bar{E}_{b} \quad$ Disturbance matrix for discretised state space building model of building $b[1 / \mathrm{s}]$

$E_{\text {total }} \quad$ Normalised total energy consumption of the building district [kWh/day/building]

$\delta \quad$ Tolerance for which price coordinator has satisfied the power supply limit

$\lambda \quad$ Price controller increasing gain parameter [-]

$\rho_{\text {air }} \quad$ Density of air $\left[\mathrm{kg} / \mathrm{m}^{3}\right]$

A Overall heat transfer area of building in dynamic building model $\left[\mathrm{m}^{2}\right]$

$C p_{\text {air }} \quad$ Specific heat capacity of air in dynamic building model $[\mathrm{kJ} /(\mathrm{kg} \mathrm{K})]$

$j^{\max } \quad$ Maximum number of iterations that the price coordinator can perform at any time step $k[-]$

$K \quad$ Price controller gain $[£ / k W h]$

$M C p_{B} \quad$ Building thermal mass in dynamic building model $[\mathrm{kJ} / \mathrm{K}]$

$n_{t} \quad$ Number of time steps in simulation [-]

$n_{a c} \quad$ Rate of air changes in dynamic building model $[1 / \mathrm{s}]$

$p^{\text {lin }} \quad$ Initial value of electricity price penalised linearly in the MPC objective function [ $\mathrm{f} / \mathrm{kWh}$ ]

$p_{k}^{\text {lin }} \quad$ Electricity price penalised linearly in the MPC objective function at simulation time step $k$ [f/kWh]

$p^{\text {quad }} \quad$ Initial value of electricity price penalised quadratically in the MPC objective function before any modification by the price coordinator $[£ / k W h]$

$p_{i \mid k}^{\text {lin }} \quad$ Electricity price at time step $i$ in the MPC prediction horizon given simulation time step $k$, penalised linearly in the MPC objective function $[£ / \mathrm{kWh}]$

Q Penalty weighting of $\epsilon_{b, i \mid j, k}^{2}$ in MPC objective function $\left[£ / \mathrm{siK}^{2}\right]$

$R \quad$ Penalty weighting of $p_{i \mid j, k}^{q u a d} u_{b, i \mid j, k}^{2}$ in MPC objective function $[1 / \mathrm{kWh}]$

Penalty weighting of $p_{i \mid k}^{\text {lin }} u_{b, i \mid j, k}$ in MPC objective function [-]
$T_{S} \quad$ Sampling time used for discretised building model [s]

$U \quad$ Overall heat transfer coefficient of building in dynamic building model $\left[\mathrm{kW} /\left(\mathrm{m}^{2} \mathrm{~K}\right)\right]$

$u^{\max } \quad$ Power supply limit or upper bound on $u_{i \mid j, k}^{\text {sum }}[\mathrm{kW}]$

$u_{b}^{U B}, u_{b}^{L B} \quad$ Heat pump load limits or upper and lower bounds respectively on $u_{b, i l j, k}$ for building $b[\mathrm{~kW}]$

$V \quad$ Total volume of building in dynamic building model $\left[\mathrm{m}^{3}\right]$

$x_{b, i \mid k}^{U B}, x_{b, i \mid k}^{L B}$ Thermal comfort boundaries or upper and lower bounds respectively on $x_{b, i l j, k}$ for building $b$ at time step $i$ in the MPC prediction horizon given the simulation time step $k[\mathrm{~K}]$

$x_{b, k}^{U B}, x_{b, k}^{L B} \quad$ Thermal comfort constraints or upper and lower bound respectively on $x_{b, k}$ for building $b$ at simulation time step $k[\mathrm{~K}]$

CBV Normalised comfort boundaries violation of the building district [C hour/day/building]

COP Coefficient of performance in dynamic building model [-]

PSLV Normalised power supply limit violations of the building district [kWh/day/building]

Variables

$\epsilon_{b, i l j, k} \quad$ Slack variable of comfort bound constraints for building $b$ at time step $i$ in the MPC prediction horizon given price iteration $j$ and simulation time step $k[\mathrm{~K}]$

$p_{k}^{\text {quad }} \quad$ Electricity price penalised quadratically in the MPC objective function at simulation time step $k[£ / k W h]$

$P_{\text {elec }} \quad$ Electrical power drawn by heat pump in dynamic building model $[\mathrm{kW}]$

$p_{i \mid j, k}^{q u a d} \quad$ Electricity price at time step $i$ in the MPC prediction horizon given price iteration $j$ and simulation time step $k$, penalised quadratically in the MPC objective function $\left[£ / \mathrm{kWh}^{2}\right]$

$Q_{c d} \quad$ Conduction term in dynamic building model $[\mathrm{kW}]$

$Q_{c v} \quad$ Convection term in dynamic building model [kW]

$Q_{g n} \quad$ Heating term in dynamic building model [kW]

$T_{\text {ext }} \quad$ External temperature in dynamic building model [K]

$T_{\text {in }} \quad$ Internal temperature of building in dynamic building model $[\mathrm{K}]$

$u_{k}^{\text {sum }}$ Total power drawn by all the heat pumps in the building network at simulation time step $k[\mathrm{~kW}]$

$u_{b, i l j, k} \quad$ Power drawn by heat pump in building $b$ at time step $i$ in the MPC prediction horizon given price iteration $j$ and simulation time step $k[\mathrm{~kW}]$

$u_{b, k} \quad$ Electrical drawn by heat pump in building $b$ at time step $k[\mathrm{~kW}]$

$u_{b \mid j, k}^{*} \quad$ Optimal power drawn by heat pump in building $b$ over the MPC prediction horizon given price iteration $j$ and simulation time step $k[\mathrm{~kW}]$

$u_{i \mid j, k}^{\text {sum }} \quad$ Total power drawn by the heat pumps in the building network at time step $i$ in the MPC prediction horizon given price iteration $j$ and simulation time step $k[\mathrm{~kW}]$

$u_{i \mid k}^{\text {sum }} \quad$ Total power drawn by the heat pumps in the building network at time step $i$ in the MPC prediction horizon given simulation time step $k[\mathrm{~kW}]$

$u_{k}^{*} \quad$ Optimal power drawn by all heat pumps in building network over the CMPC prediction horizon at simulation time step $k[\mathrm{~kW}]$ 


\section{$u_{k}^{\text {sum }} \quad$ Total power drawn by heat pumps in building net- work at time step $k[\mathrm{~kW}]$ \\ $x_{b, i j, k} \quad$ Internal temperature of building $b$ at time step $i$ in the MPC prediction horizon given price iteration $j$ and simulation time step $k[\mathrm{~K}]$ \\ $x_{b, k} \quad$ Internal temperature of building $b$ at time step $k[\mathrm{~K}]$}

to the system, and by repeating the process at the next time instance feedback is introduced into the control system. Economic MPC (EMPC) differs from traditional MPC in the optimisation criteria - instead of minimising deviation from a set point, the total costs over the time horizon are minimised while the system outputs are required to stay within specified bounds.

While a review by Afram and Janabi-Sharifi [7] found that most papers on MPC and HVAC control used a classical tracking formulation, EMPC has nonetheless proved popular in HVAC Control. Zong et al. [8] employed an EMPC to control zones in a building with the scope of using it as a demand side management tool in a smart grid, while [9] used EMPC to take advantage of a thermal energy storage unit. One of the challenges of EMPC is that due to the linear cost function, the resulting control strategy can be erratic in response to a time varying electricity price. For this reason [10] developed an infinite horizon formulation of the EMPC problem to deal with the sharp peaks in the system input.

\subsection{Coordinated building control}

Often research into HVAC control involves buildings with several climatic zones [11], district heating networks supplying multiple buildings [12] or micro grids in which scheduling of energy sources is carried out while satisfying the buildings' heating and electricity demands [13]. These separate subsystems interact with each other, for example through thermal coupling in the case of zones in a building or through limits on the resources that a building can consume in a district heating network. Adopting a centralised approach such as in [14] can adequately account for interactions between subsystems, since a centralised controller has all the information required to control subsystems while respecting constraints. This is not possible in an uncoordinated setup, where each subsystem has its own controller which behaves independently of how it affects and is affected by neighbouring subsystems. A centralised controller is however computationally expensive and less reliable, since any problem in the controller will disable the entire control system [7]. Additionally, there are privacy concerns since potentially sensitive data about residents is collected centrally and stored [15].

Distributed algorithms use communication between subsystem controllers in order to account for subsystem interactions and converge towards a globally optimal solution, with the advantage of increased reliability and a computational burden that does not increase exponentially with the problem size. Morosan et al. [11] uses distributed MPC (DMPC) to account for thermal coupling between zones, while in Scherer et al. [16] the interaction is due to limits on the resources available to the building, the zones being considered to be thermally isolated from each other. This limit on resources was introduced in the model due to renewable energy sources, whose intermittent nature led to the energy requirements of the building needing coordination to be fulfilled. In contrast, Lamoudi et al. [17] use a bundle method to deal with similar restrictions on building resource consumption, while [13] present a generic DMPC framework for use in microgrids to coordinate distributed energy resources.

Another common coordination approach is hierarchical control, whereby a decomposition of the optimal control strategy into two (or more) levels allows for an increase in reliability and reduction in computation time of the optimal control strategy for a large system. The levels in the hierarchy interact only with the levels directly above and below it, unlike in a distributed control framework where a hierarchy may exist only to allow information sharing between subsystem controllers. One of the disadvantages of some distributed frameworks is the number of iterations and therefore information exchanges which are required, since existing HVAC communication protocols are limited. Rawlings et al. [18] therefore proposes a hierarchical framework for large HVAC systems, where the solution to a high-level problem which considers the entire system in approximate form is used to solve two low-level subproblems that consider the airside and waterside subsystems in more detail. Wu et al. [12] and Parisio et al. [19] both use a hierarchical framework in the context of microgrids, with the former using it for better scheduling of combined heat and power units and the latter for coordination between a group of microgrids.

The above papers approach coordination from a control perspective. The problem can also be formulated as a question of determining the price of resources which achieve coordination, e.g. to reduce peak demands. For example, Li et al. [20] determines the time varying electricity prices which maximise social welfare, that is total customer spending minus the utility company's cost of providing electricity, for a group of households.

This paper combines the control and economic perspectives by coordinating distributed MPCs using a price signal. To the authors' best knowledge, there is no literature which investigates the proposed hierarchical approach, where a top level controller coordinates multiple buildings' heat pump controllers using a price signal. It is worth noting that [21] does use a similar coordination strategy, the Alternating Direction Method of Multipliers (ADMM) algorithm, to that presented here, though this is done on a day ahead and not rolling horizon basis and with a focus on demand side management.

\subsection{Scope and structure}

In this paper, a hierarchical control method is used to coordinate a network of buildings using heat pumps in the presence of a limit on the total power that can be drawn by said heat pumps. The proposed coordination method is able to reduce peak power demands below this limit, which could arise for a variety of reasons; the local utility could experience supply issues for example, or the network may be connected to a microgrid with a significant limit on the electrical power that it can provide, as in [16]. Sophisticated control strategies such as the one presented here, which are able to influence when power is consumed i.e. act as a demand response mechanism, are also useful in order for the electrical grid to actively respond to the constraints imposed by the increased penetration of renewable energy sources [22, p. 212].

In the proposed framework, each building in the network has its heat pump controlled by an MPC minimising total comfort bound violations, electricity costs and a pseudo electricity cost over its prediction horizon. The MPCs send their predicted system inputs (i.e. power drawn by the building's heat pump) to a higher level price coordinator. This price coordinator increases the pseudo electricity price at points in the prediction horizon where the total power drawn by the heat pumps is greater than the power supply limit. This price signal is sent to the MPCs which update their predicted inputs accordingly and the process is repeated until the power supply limit is no longer breached. A previous study determined that MPCs which penalise the electricity costs linearly led to instability issues, and hence in this work an increase in a pseudo electricity price penalised quadratically in the MPC objective function was used to achieve coordination [23]. The proposed 
method is attractive because it is a simple and intuitive way of approaching coordination in this context: simple because only predicted power demand and a pseudo electricity price are exchanged between the price coordinator and the network, and intuitive since peaks in power demand are discouraged by increasing this pseudo price of electricity.

This paper is structured as follows. Section 2 presents the control methodology used in this study. Section 3 presents the building model and building networks used for simulations. Results are shown in Section 4, with Section 4.1 presenting an analysis of the price coordinator's behaviour while Section 4.2 presents a 100 building network case study to compare the performance of the price coordinator with that of a centralised MPC controller. A discussion of the results and simulations is presented in Section 4.3 along with suggestions for further work. Lastly, Section 5 presents conclusions.

\section{Control methodology}

\subsection{MPC formulation}

The proposed price coordinator approach to coordination requires a network of buildings whose heat pumps are controlled by MPCs which interact iteratively with the price controller. For each building $b \in \mathcal{B}$, the $j$ th iteration of the objective and constraints with prediction horizon $N$ at time-step $k$, can be given as:

$u_{b \mid j, k}^{*}=\underset{\epsilon_{b, i \mid j, k}, u_{b, i \mid j, k}, x_{b, i \mid j, k}}{\arg \min } \sum_{i=0}^{N-1} Q \epsilon_{b, i+1 \mid j, k}^{2}+R p_{i \mid j, k}^{q u a d} u_{b, i \mid j, k}^{2}+r p_{i \mid k}^{l i n} u_{b, i \mid j, k}$

s.t.

$x_{b, i=0 \mid j, k}=x_{b, k}$

$x_{b, i+1 \mid j, k}=\bar{A}_{b} x_{b, i \mid j, k}+\bar{B}_{b} u_{b, i \mid j, k}+\bar{E}_{b} d_{i \mid k}$

$x_{b, i+1 \mid j, k}^{L B}-\epsilon_{b, i+1 \mid j, k} \leq x_{b, i+1 \mid j, k} \leq x_{b, i+1 \mid j, k}^{U B}+\epsilon_{b, i+1 \mid j, k}$

$u_{b}^{L B} \leq u_{b, i \mid j, k} \leq u_{b}^{U B}$

$\epsilon_{b, i \mid j, k} \geq 0$

$\forall i \in[0, N-1]$

At every time step $k$ and price iteration $j$, the MPC controller solves the above optimisation problem to determine the optimal system input over the time horizon, $u_{b, i \mid j, k}^{*} \forall i \in[0, N-1]$. The first of these inputs, $u_{b, i=0 \mid j, k}^{*}$ is applied to the system, the controller waits to observe the next initial point $x_{b, k+1}$ and the process is repeated. In the absence of a price coordinator, the index $j$ is unnecessary.

For building $b$ at simulation time step $k$ the state variable $x_{b, k}$ is the internal temperature of the building, the system input $u_{b, k}$ is the electrical power drawn by the heat pump controlled by the MPC and the system disturbance $d_{k}$ is the external temperature. The slack variable $\epsilon_{b, k}$ represents the amount that a comfort bound has been violated in ${ }^{\circ} \mathrm{C}$.

The objective function contains 3 terms summed over the prediction horizon, with weightings $Q R$ and $r$ which, for simplicity of analysis of the price coordinator, were kept the same for all the buildings in the network. The first term, $Q \epsilon_{b, i \mid j, k}^{2}$ penalises violations of comfort bounds during the prediction horizon, since Eq. (4) forces $\epsilon_{b, i l j, k}$ to be non negative for any value of $x_{b, i l j, k}$ outside the comfort bounds given by the interval $\left[x_{b, i \mid k}^{L B}, x_{b, i \mid k}^{U B}\right]$. These soft constraints on the internal building temperature are required to ensure feasibility and to allow the price coordinator to force violations of the comfort bounds if needed, so as to satisfy the hard supply constraints. It was chosen to penalise the slack variables quadratically since this better reflects consumer preferences, with small temperature deviations being negligible but larger ones unacceptable. The downside to a quadratic instead of linear penalty is that the latter could be interpreted as the cost per unit temperature violated that the consumer is willing to accept. As it is, $Q$ has nominal units of $£ / K^{2}$ and therefore no physical significance.

The second term in the objective function, $R p_{i \mid j, k}^{q u a d} u_{b, i \mid j, k}^{2}$, penalises the electrical power usage quadratically at step $i$ in the prediction horizon if $p_{i \mid j, k}^{q u a d}$, referred to in this paper as the quadratic electricity price, is greater than zero. The price coordinator influences the MPCs control strategy by increasing the price to discourage power usage at that step in the prediction horizon. In this paper the quadratic electricity price was treated merely as a tool for coordination and not a real electricity price, since the values of $p_{i \mid j, k}^{q u a d}$ were determined entirely by the price coordinator and therefore had no economic basis.

The last term in the objective function, $r p_{i \mid k}^{l i n} u_{b, i \mid j, k}$, minimises total electricity costs by penalising the system inputs linearly, and hence $p_{i \mid k}^{l i n}$ is referred to here as the linear electricity price. This term is also said to minimise total energy consumption in this study since $p_{i \mid k}^{\text {lin }}$ was kept constant, in which case cost and energy minimisation are equivalent. This term is present since the overall strategy for the price based coordination is to initially have each household MPC minimise total costs, which is desirable from the consumer's perspective, and only if the power supply limit is violated does the price coordinator step in, since its' objective is peak reduction which is necessary for the electrical power system to function. Choosing the weightings $Q R$ and $r$ represent a trade off therefore between minimising comfort boundary violations, peaks in power demand and total electricity costs.

For the rest of the formulation, Eq. (2) represents the initial condition, which is assumed to be known exactly. Eq. (3) describes dynamics of the model which are detailed in Section 3.1, for which perfect predictions of the disturbance $d_{i \mid k} \forall i \in[0, N-1]$ are assumed. Eq. (4) describes the internal temperature soft constraints as noted before, while Eq. (5) describes the bounds on the electrical power that the building's heat pump can draw. These are inviolable and time independent. Finally, Eq. (6) ensures that the slack variables are non-negative.

\subsection{Centralised MPC formulation}

In the case of centralised control, the individual building MPCs are replaced by a single MPC (CMPC) which controls the internal temperatures of all the buildings in the network. This centralised framework was used as a baseline with which to compare the performance of the price coordinator.

At each time step $k$, the CMPC minimises the cost function shown in Eq. 7 while respecting the constraints from Eqs. (2)-(6) for $b \in \mathcal{B}$ and omitting the price iteration index $j$, since there is no price controller in the centralised case. The additional power supply limit constraint shown in Eq. (8) is also included.

$$
\begin{aligned}
& u_{k}^{*}=\underset{\epsilon_{b, i \mid k}, u_{b, i \mid k}, x_{b, i \mid k}}{\arg \min } \sum_{b \in \mathcal{B}} \sum_{i=0}^{N-1} Q \epsilon_{b, i+1 \mid k}^{2}+r p_{i \mid k}^{l i n} u_{b, i \mid k} \\
& u_{i \mid k}^{\text {sum }} \leq u^{\max } \forall i \in[0, N-1]
\end{aligned}
$$

where $u_{i \mid k}^{\text {sum }}$ is the total electrical power drawn by the heat pumps in the building network defined by Eq. (9), also referred to as the 


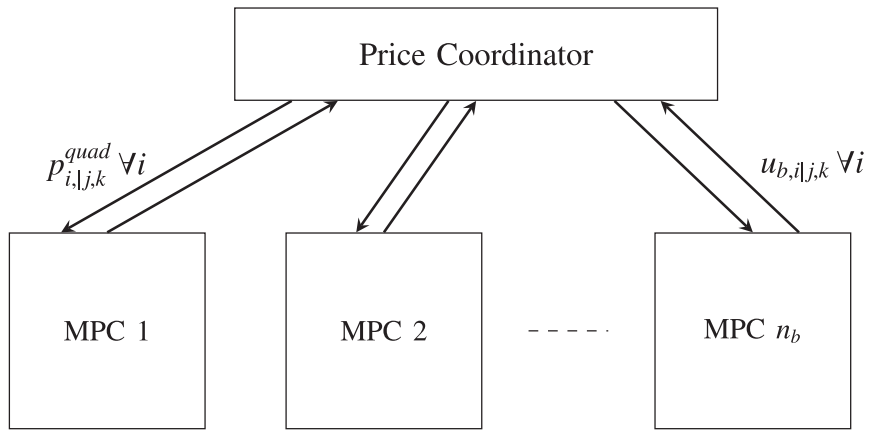

Fig. 1. Schematic of price coordinator control structure with information flows shown for price iteration $j$ and simulation time step $k$.

load profile of the network or simply the load profile. ${ }^{1}$

$u_{i \mid k}^{\text {sum }}=\sum_{b \in \mathcal{B}} u_{b, i \mid k}$

As in the case for the individual building MPC, the first system input $u_{b, i=0 \mid k}^{*}$ is applied for each building $b \in \mathcal{B}$, the CMPC records the next state $x_{b, i \mid k+1}$, uses this as an initial point to solve the above optimisation problem and so the process is repeated. By knowing the predicted electrical power drawn by all the heat pumps in the network, $u_{b, i \mid k} \forall b \in \mathcal{B}$, the CMPC is able to respect the power supply limit constraint represented by Eq. (8). This ensures that $u_{i \mid k}^{\text {sum }}$ stays below the power supply limit $u^{\max }$ at all times during the simulation. There is therefore no need for the quadratic electricity price $p_{i \mid j, k}^{q u a d}$ in the CMPC formulation.

\subsection{Hierarchical price coordinator}

In the absence of a centralised controller, scenarios can arise whereby the building network's requested electrical load exceeds limitations of the supply networks. Indeed this was the motivation behind the use of a CMPC, which is able to respect this limitation by introducing the constraint shown in Eq. (8) into its optimisation problem. As mentioned in Section 1.3, the CMPC suffers from computational, reliability and privacy issues, hence the need for alternative coordination strategies such as the price coordinator presented here.

The hierarchical price controller proposed here seeks to replicate the performance of the centralised approach without such issues while satisfying the constraint of Eq. (8). When the buildinglevel supply requests violates the power supply limit (see Eq. (8)), the price coordinator penalises the MPC controllers by increasing the price of electricity quadratically by an amount proportional to the violation. See Fig. 1 for a graphical representation of this process between the price coordinator and the MPCs in the network. Pseudocode representing the price coordinator algorithm is shown in Algorithm 1.

The pricing coordinator was based on a classical proportional controller as shown in Algorithm 1. It receives as inputs the building network's predicted load profile $u_{i \mid j, k}^{s u m}$ (see Eq. (10)), the total capacity of the heat pumps in the building network $u^{\text {sum,UB }}$ as defined by Eq. (11), the power supply limit $u^{\max }$, the current quadratic electricity price over the prediction horizon $p_{j, i \mid k}^{q u a d}$ and the current price iteration $j$.

\footnotetext{
1 Calling this a load profile is not technically correct, since in reality the building network would have an electrical load which includes consumption due to e.g. lighting as well as the heat pumps. However, only the load due to the heat pumps is considered in this paper, and hence for the sake of brevity load profile is also used.
}

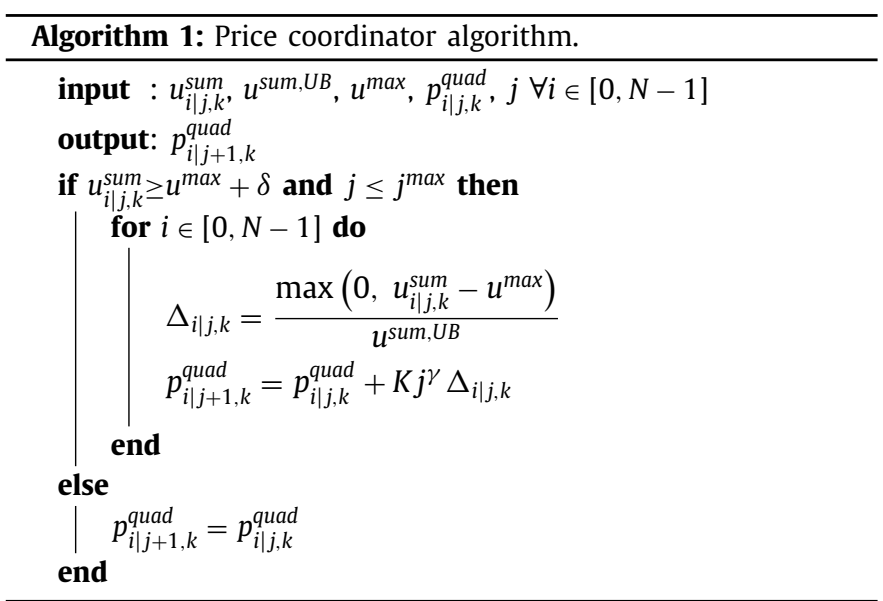

$u_{i \mid j, k}^{\text {sum }}=\sum_{b \in \mathcal{B}} u_{b, i \mid j, k}$

$u^{s u m, U B}=\sum_{b \in \mathcal{B}} u_{b}^{U B}$

It checks whether the power supply limit is violated within some tolerance $\delta$ or the maximum number of iterations has been reached before proceeding. If either of these criteria are not met, the price coordinator does nothing and the last optimal computed system input $u_{b, i=0 \mid j, k}^{*} \forall b \in \mathcal{B}$ is applied to the buildings by their respective MPC controllers and the simulation moves one time step, $k=k+1$. $\delta$ was chosen to be $0.1 \% u^{\text {sum,UB }}$, as shown in Eq. (12).

$\delta=\frac{u^{\text {sum }, U B}}{1000}$

If the aforementioned criteria are met, the price coordinator calculates the violation of the supply limit by taking the difference between $u_{i, j \mid k}^{\text {sum }}$ and $u^{\max }$, applying the ramp function to it and then normalising this by $u^{\text {sum,UB }}$ as in Eq. (13).

$\Delta_{i \mid j, k}=\frac{\max \left(0, u_{s u m}-u_{\max }\right)}{u^{\text {sum }, U B}}$

The algorithm output $p_{i \mid j+1, k}^{q u a d}$ is then calculated as the previous electricity price $p_{i \mid j, k}^{q u a d}$ plus the violation of the power supply limit multiplied by some gain $\mathrm{Kj}^{\gamma}$ as shown in Eq. (14).

$p_{i \mid j+1, k}^{q u a d}=p_{i \mid j, k}^{q u a d}+K j^{\gamma} \Delta_{i \mid j, k}$

$K$ is static, while the term $j^{\gamma}$ increases with $j$. This last feature of the algorithm is introduced in the expectation that if initially $p_{i \mid j, k}^{q u a d}$ is not increased enough to alter the MPCs' behaviour, further iterations will lead to $\mathrm{Kj}^{\gamma}$ increasing enough to achieve this. The low value of $\gamma$ and $j^{\max }$ used in this study meant that this increase was limited to approximately $20 \times K$.

The linear electricity price was kept constant for all simulations with a value of $p_{k}^{\text {lin }}=0.1 / k W h \forall k$ (except for in Section 4.1.1), while the price coordinator parameters were selected such that $p_{i \mid j, k}^{q u a d}$ increased by less than $0.1 \mathrm{f} / \mathrm{kWh}$ per iteration. The behaviour of the MPCs depends on the relative ratios of $Q R, r, p_{i \mid j, k}^{q u a d}$ and $p_{i \mid k}^{l i n}$, and hence the particular values assigned to $p_{k}^{\text {lin }} \forall k$ considered in isolation are somewhat arbitrary.

In this paper, the price coordinator increases $p_{i \mid j, k}^{\text {quad }}$ instead of $p_{i \mid j, k}^{l i n}$ due to the convergence issues noted in [23]. This instability occurred because optimisation problems with a linear objective function are not smooth like quadratic ones, and so small changes 
in the electricity price lead to the optimal solution jumping from one boundary to another with no reduction in $\Delta_{i \mid j, k}$. Strbac and Kirschen [22] illustrate this problem in a similar context to the one studied here.

\section{Simulated building network development}

To analyse the price coordinator's behaviour, a building network $^{2}$ was required with a power supply limit dictating the maximum electrical power that could be drawn by the heat pumps in the network. For this purpose, Sections 4.1.2 to 4.1.4 used a simple 2 building network to analyse the price coordinator's behaviour. A 100 building network was then simulated in Section 4.2 as a proof of concept and to compare simulation results with that of a network controlled by a CMPC.

\subsection{Building model}

As the performance of the price coordination strategy is the focus of this study, the simple building model shown below in Eqs. (15) to (18) was chosen. This model could be further built upon, for example by considering internal and external gains as done by Oldewurtel et al. [24], but these were not considered in this work.

$Q_{c d}=\left(T_{i n}-T_{e x t}\right) \cdot U \cdot A$

$Q_{c v}=\left(T_{\text {in }}-T_{\text {ext }}\right) \cdot \rho_{\text {air }} \cdot V \cdot C p_{\text {air }} \cdot n_{a c}$

$\operatorname{MCp}_{B} \dot{T_{i n}}=Q_{g n}-Q_{c d}-Q_{c v}$

$Q_{g n}=P_{\text {elec }} \cdot \mathrm{COP}$

Eq. (15) expresses the conduction term $Q_{c d}$ as the overall heat transfer coefficient $U$ (defined for the entire building) multiplied by the area available for heat transfer $A$ and the internal and external temperature difference $\left(T_{i n}-T_{\text {ext }}\right)$. Eq. (16) expresses the convection term $Q_{c v}$ as the number of air changes per hour $n_{a c}$ multiplied by the difference in energy content between the inside and outside air. Eq. (17) is the dynamic energy balance of the building where $M C p_{b}$ is the building thermal mass and $Q_{g n}$ is the heat input into the building. $Q_{g n}$ is related to the system input, the electrical power drawn by the heat pump $P_{\text {elec }}$, through the heat pump COP (assumed fixed) as expressed in Eq. (18).

This system can be represented as a single input single output (SISO) state space model with state $T_{i n}$, input $P_{\text {elec }}$ and disturbance $T_{\text {ext }}$ as shown in Eqs. (19) through (22) (note that Kelvin must be used instead of Celsius in this case). The state, input and disturbance variables have been changed to $x, u$ and $d$ to be consistent with standard state space notation, with the subscript $b$ indicating that these parameters are specific to the building $b$ 's characteristics.

$\dot{x}=A_{b} x+B_{b} u+E_{b} d$

$A_{b}=\frac{U \cdot A+\rho_{a i r} \cdot V \cdot C p_{a i r} \cdot n_{a c}}{M \cdot C p_{B}}$

$B_{b}=\frac{\mathrm{COP}}{M \cdot C p_{B}}$

$E_{b}=-\frac{U \cdot A+\rho_{a i r} \cdot V \cdot C p_{a i r} \cdot n_{a c}}{M \cdot C p_{B}}$

Since the MPC requires a discretised model of the system, the above state space model was then discretised using the zero-order

\footnotetext{
${ }^{2}$ In this paper a building network simply refers to a group or cluster of buildings.
}

Table 1

Summary of parameter values used for building models.

\begin{tabular}{llll}
\hline Building type & 1BF_H & 2BF_H & 3BF_H \\
\hline Heat exchange surface area $A\left[\mathrm{~m}^{2}\right]$ & 42.48 & 54.13 & 82.07 \\
Mean $U$ value $\left[\mathrm{kW} /\left(\mathrm{m}^{2} \mathrm{~K}\right)\right]$ & 0.93 & 0.93 & 0.93 \\
Building volume $V\left[\mathrm{~m}^{3}\right]$ & 115.8 & 147.8 & 224.1 \\
Air changes per second $n_{a c}[1 / \mathrm{s}]$ & 3.600 & 3.600 & 3.600 \\
Air density $\rho_{\text {air }}\left[\mathrm{kg} /\left(\mathrm{m}^{3}\right)\right]$ & 1.23 & 1.23 & 1.23 \\
Air heat capacity $C p_{a i r}[\mathrm{~kJ} /(\mathrm{kgK})]$ & 1.01 & 1.01 & 1.01 \\
Building thermal mass $M C \cdot p_{b}[\mathrm{~kJ} / \mathrm{K}]$ & 7.901 & 36,681 & 15,287 \\
Heat pump capacity $u^{U B}[\mathrm{~kW}]$ & 12 & 12 & 12 \\
Heat pump COP $[-]$ & 2.5 & 2.5 & 2.5 \\
\hline
\end{tabular}

hold method and a sampling time $T_{S}$ of 15 min since this is 20 to 30 times shorter than the settling time of any of the buildings considered. The discretised model is written as shown in Eq. (23).

$x_{k+1}=\bar{A}_{b} x_{k}+\bar{B}_{b} u_{k}+\bar{E}_{b} d_{k}$

\subsection{Simulation parameters}

The parameters used for the building model were determined by conducting a survey to estimate relevant geometric and thermophysical coefficients for typical UK residential buildings and then calculating thermal mass. A detailed account of this survey can be found in [25], the result of which was a library of parameters representing various housing types (e.g. semi-detached, flat, house etc). Table 1 lists the building types that were used for the building network case studies. In Section 4.1, the 2 buildings used were 3BF_H (3 bedroom flats with high energy consumption), while the 100 building network considered in Section 4.2 consisted of a uniform mix of all 3 building types shown in Table 1 such that the building network could represent a block of flats for example.

In all simulations the comfort bounds were 22 to $25^{\circ} \mathrm{C}$ during occupancy and 16 to $30^{\circ} \mathrm{C}$ during non occupancy. For the 2 building analysis in Section 4.1, occupancy was from 6 to 9 AM and 6 to $10 \mathrm{PM}$ for both buildings. For the 100 building case study in Section 4.2 occupancy profiles were obtained by the method outlined in [26].

The external temperatures used in simulations were those of West London, UK for the month of January 2014, with simulations starting at midnight. Note that communication interfaces between the MPCs and the price coordinator were not considered, though this topic and its difficulties has been broached elsewhere (see Fischer and Madani [27]).

For simplicity, the weighting matrices $Q R$ and $r$ were the same for every building in the network, as was the sampling time $T_{S}$ and prediction horizon $N$, which was chosen to be a day. For the implementation of the MPC algorithm, if the optimisation problem failed at time step $k$ and price iteration $j=j^{\max }$ (which occurred for some realisations of the price signal), the last optimal input was applied to the system, i.e. $u_{b, i=0 \mid j, k}^{*} \forall b \in \mathcal{B}$ for the last $j$ for which an optimal solution was found, before continuing. All simulations were performed using MATLAB R2017a and the quadprog solver.

\subsection{Performance metrics}

To compare results from the simulations, several key performance metrics were used as listed below. All of these metrics are calculated over the simulation time, that is $\forall k \in\left[0, n_{t}\right]$ where $n_{t}$ is the number of time steps of the simulation. They are then normalised by the simulation time in days and number of buildings $n_{b}=|\mathcal{B}|$ in the network. Note that $R(z)=\max (0, z)$ is the ramp function. 
Total energy consumption. Defined as $\frac{k W h / d a y}{\text { building }}$. This is a measure of the energy efficiency of a control strategy. Note that since in this paper $p_{k}^{\text {lin }}$ is constant $\forall k$ this is equivalent to the total electricity cost over the simulation. However since using electricity costs as a metric could cause confusion due to the quadratic electricity price, total energy consumption was used instead.

$\mathrm{E}_{\text {total }}=\left(\sum_{k=0}^{n_{t}} \sum_{b \in \mathcal{B}} u_{b, k}\right) T_{s} \frac{(24 \cdot 60 \cdot 60)}{n_{t} T_{s} n_{b}}$

Comfort boundaries violations. Defined as $\frac{{ }^{\circ} \mathrm{C} \text { hour/day }}{\text { building }}$. This is a measure of how well a control strategy satisfied comfort constraints (i.e. the bounds on internal temperature). Often (though not necessarily) an increase in thermal comfort (decrease in CBV) comes at the cost of a decrease in energy efficiency (increase in $\mathrm{E}_{\text {total }}$ ).

$\mathrm{CBV}=\left(\sum_{k=0}^{n_{t}} R\left(x_{b, k}-x_{b, k}^{U B}\right)+R\left(x_{b, k}^{L B}-x_{b, k}\right)\right) T_{s} \frac{(24 \cdot 60 \cdot 60)}{n_{t} T_{s} n_{b}}$

Power supply limit violations. Defined as $\frac{k W h / d a y}{\text { building }}$ This is a measure of how good the coordination strategies considered here (centralised and price coordinator) are. For the centralised method this measure should be 0 since Eq. (8) is a hard constraint. $u_{k}^{\text {sum }}$ is given by Eq. (27).

$\operatorname{PSLV}=\sum_{k=0}^{n_{t}}\left(R\left(u_{k}^{\text {sum }}-u_{\max }\right)\right) T_{s} \frac{(24 \cdot 60 \cdot 60)}{n_{t} T_{s} n_{b}}$
$u_{k}^{\text {sum }}=\sum_{b \in \mathcal{B}} u_{b, k}$

\section{Results}

\subsection{Analysis and performance evaluation of price coordination strategy}

In this Section the price coordinator's behaviour was analysed. This was first done by exploring at the MPC's control strategy for linear and quadratic penalties on the system input $u_{b, i \mid k}$ in Section 4.1.1 without any price coordination. In Section 4.1.2 a 2 building network was used to determine for what values of $Q / R$ comfort bounds were violated, again without price coordination. Section 4.1.3 compares price coordination with CMPC control for the 2 building case and illustrates the negotiation between the price controller and the building network MPCs. Finally a Pareto analysis in Section 4.1.4 investigates the trade off between comfort violations, energy consumption and peak reduction in the presence of different power supply limits.

\subsubsection{Comparison of control strategy for linear and quadratic price penalties for a single building without price coordination}

In the price coordination strategy employed here, a purely linear price penalty in the MPC objective function is used to minimise energy during normal operation while a quadratic penalty is needed to reduce peaks when the power supply limit is violated. To investigate the effect these different penalties have on the MPC's control strategy, the values of $R$ and $r$ were varied for a fixed $Q$ and a constant linear and quadratic electricity price. This was done for a single building and $Q=1000$ to ensure that the comfort bound violations are heavily penalised, with the result shown in Fig. 2. Parameters for this simulation are shown in Table 2.

With reference to Fig. 2, in the instance where only a linear price penalty is present $(R=0, r=1) u_{b, k}$ is mostly 0 when the comfort bounds are relaxed. There is then a sharp increase which reaches $u_{b}^{U B}$ just before these comfort bounds are tightened. When
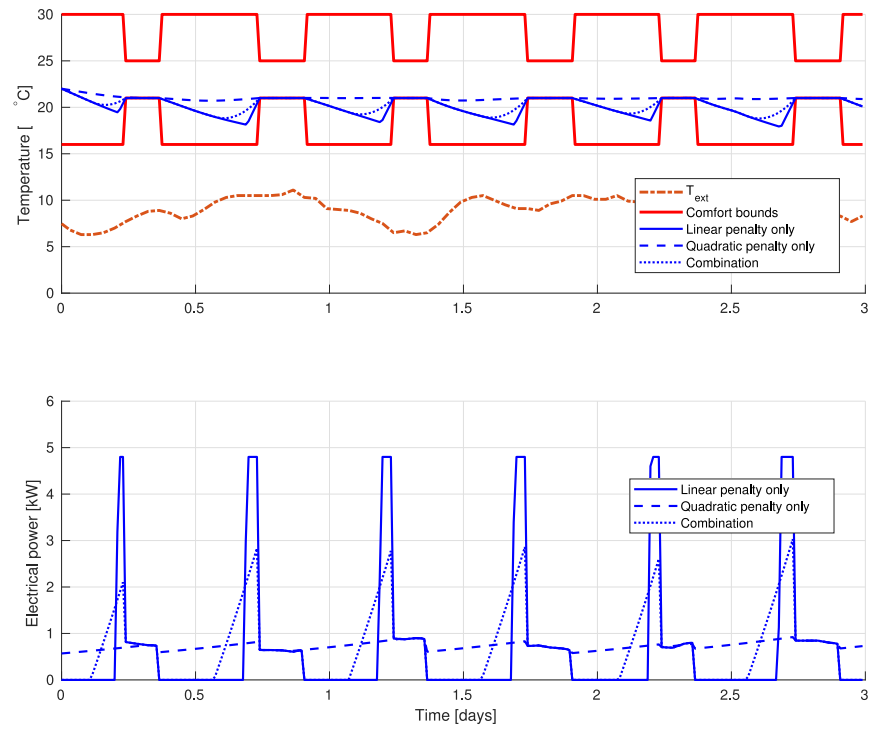

Fig. 2. MPC control strategy for linear, quadratic and a combination of penalties on the inputs. Top figure shows comfort bounds and internal and external temperatures while the bottom figure is the electrical power drawn by the heat pumps.

Table 2

Simulation parameters for the comparison of control strategies in the presence of linear and quadratic price penalties for a single building without price coordination.

\begin{tabular}{|c|c|c|c|}
\hline \multirow[t]{2}{*}{ Parameter [Units] } & \multicolumn{3}{|c|}{ Value } \\
\hline & Linear penalty only & Combined & Quadratic penalty only \\
\hline$Q\left[£ / \mathrm{siK}^{2}\right]$ & \multicolumn{3}{|c|}{1000} \\
\hline$R[1 / \mathrm{kWh}]$ & 0 & 0.05 & 1 \\
\hline$r[-]$ & 1 & 0.95 & 0 \\
\hline Simulation time [days] & \multicolumn{3}{|c|}{3} \\
\hline$N$ [\# of time steps] & \multicolumn{3}{|c|}{96} \\
\hline$\left[p^{\text {lin }}, p^{\text {quad }}\right][£ / \mathrm{kWh}]$ & \multicolumn{3}{|c|}{$[1,1]$} \\
\hline Initial temperature $\left[{ }^{\circ} \mathrm{C}\right]$ & \multicolumn{3}{|c|}{22} \\
\hline
\end{tabular}

only a quadratic price penalty is present $(R=1, r=0), u_{b, k}$ varies much less, never reaching $0 \mathrm{~kW}$ and staying below $2 \mathrm{~kW}$ at all times. In the last instance a combination of linear and quadratic price penalties is used $(R=0.05, r=0.95)$, demonstrating that a mixture of penalties leads to a spreading of the peak of $u_{b, k}$ over a longer period of time, and therefore a reduction in the magnitude of this peak. This difference in behaviour is easily explained by the fact that a high value of $u_{b, k}$ (assuming constant electricity price) is penalised much more in the quadratic than in the linear price penalty case. Since the quadratic price penalty does not minimise the total energy cost in the objective function, it is natural that this will also lead to an equal or higher value of $E_{\text {total }}$ for a constant cost. It is clear also that the choice of control strategy could reduce the size of the installed heat pump while not sacrificing user comfort.

The key insight here is that by introducing a quadratic penalty in the MPC, it is possible to reduce the peaks in $u_{b, k}$ by spreading out the energy consumed by the heat pump over a longer period of time. In the case of a network with a price coordinator, the peaks in $u_{i \mid j, k}^{s u m}$ are reduced by the price coordinator increasing $p_{b, j, i \mid k}^{\text {quad }}$ which allows the power supply limit constraint to be respected. The overall strategy for coordination of the network then is to use the linear penalty if the power supply limit is not breached since it minimises total costs or energy (they are equivalent in this case), and in the presence of a power supply limit violation the quadratic price penalty is introduced by the price coordinator to minimise the peaks, possibly at the expense of comfort boundary violations. 
Table 3

Simulation parameters for the investigation into the comfort to flexibility ratios $Q / r$ and $Q / R .8$ simulations were run in total, 4 with only linear penalties ([ $\left.p^{\text {lin }}, p^{\text {quad }}\right]$ $=[0.1,0])$ varying $Q / r$ and 4 with only quadratic penalties $\left(\left[p^{\text {lin }}, p^{q u a d}\right]=[0,0.1]\right)$ varying $Q / R$.

\begin{tabular}{|c|c|c|c|c|}
\hline \multirow[t]{2}{*}{ Parameter [Units] } & \multicolumn{4}{|c|}{ Value } \\
\hline & Scenario 1 & Scenario 2 & Scenario 3 & Scenario 4 \\
\hline$Q\left[£ / \mathrm{siK}^{2}\right]$ & \multicolumn{4}{|c|}{10} \\
\hline$R[1 / \mathrm{kWh}]$ & 1 & 10 & 100 & 1000 \\
\hline$r[-]$ & 1 & 10 & 100 & 1000 \\
\hline Simulation time [days] & \multicolumn{4}{|c|}{3} \\
\hline$N$ [\# of time steps] & \multicolumn{4}{|c|}{96} \\
\hline$\left[p^{\text {lin }}, p^{\text {quad }}\right][£ / \mathrm{kWh}]$ & \multicolumn{4}{|c|}{$Q / r:[0.1,0], Q / R:[0,0.1]$} \\
\hline Initial temperature $\left[{ }^{\circ} \mathrm{C}\right]$ & \multicolumn{4}{|c|}{22} \\
\hline
\end{tabular}

Table 4

Comfort boundary violations (CBV) for varying comfort to flexibility ratios $Q / r$ and $Q / R$.

\begin{tabular}{lllll}
\hline$Q / r$ or $Q / R$ & 10 & 1 & 0.1 & 0.01 \\
\hline CBV for $Q / r$ & 0.0136 & 0.135 & 1.34 & 13.1 \\
CBV for $Q / R$ & 0.00882 & 0.0873 & 0.876 & 10.2 \\
\hline
\end{tabular}

\subsubsection{Objective function tuning parameter considerations}

The price controller works by adding a quadratic penalty to the objective function of the MPCs, leading to a spreading of the peak of $u_{k}^{\text {sum }}$ over a longer period of time and therefore reducing the size of this peak. There could be situations where satisfying the power supply limit constraint requires the comfort boundaries to be violated as well. For this reason the ratios $Q / R$ and $Q / r$ and their effect on controller behaviour were investigated for a 2 building network without any price coordination, since these ratios determine how readily the comfort boundaries are violated. Low values of $Q / R$ in particular allow the price coordinator to influence MPC behaviour more. Simulation values are detailed in Table 3, with 8 simulations being run in total; 4 in which only linear penalties were present and $Q / r$ was varied and 4 where only quadratic penalties were present $\left(p^{\text {lin }}=0\right)$ and $Q / R$ was varied.

Comparing CBV values in Table 4 with internal temperatures in Fig. $3 \mathrm{a}$ and $\mathrm{b}$, it is clear that $\mathrm{CBV}$ values at or below $1 \frac{{ }^{\circ} \mathrm{C} \text { hour/day }}{\text { building }}$ are not significant (this is important to keep in mind when in-
Table 5

Performance metrics for simulations of 2 building network for 3 different cases Simulation times were recorded on an HP Elitebook 840 G2 with Intel Core i5$5200 \mathrm{U}$ CPU $2.20 \mathrm{GHz} 4,4 \mathrm{GiB}$ of RAM running on 64 -bit Ubuntu.

\begin{tabular}{llll}
\hline Performance Metric & Uncoordinated & Centralised & Price Coordinator \\
\hline $\mathrm{E}_{\text {total }}[\mathrm{kWh} /$ day/building] & 14.6225 & 15.0571 & 14.4825 \\
CBV [C hour/day/building] & 0.0152 & 0.0162 & 0.0153 \\
PSLV [kWh/day/building] & 6.1858 & 0 & $1.5247 \times 10^{-} 5$ \\
Simulation Time [s] & 19.1 & 21.8 & 29.7 \\
\hline
\end{tabular}

terpreting results in Sections 4.1 .3 and 4.2). Since this corresponds to a $Q / r$ or $Q / R$ ratio of $0.1, Q / r=10$ was chosen for subsequent simulations to ensure that initially the comfort bounds were strict soft constraints, while the $Q / R$ ratio was further investigated in Section 4.1.4.

\subsubsection{Simulation of 2 building network with price coordination}

To demonstrate the price coordinator in action, the 2 building network considered in Section 4.1.1 is simulated here for 3 cases:

- Uncoordinated - No interaction between the MPCs in different buildings, and hence possible violation of the power supply limit

- Centralised - The CMPC outlined in Section 2.2 is used to control the entire building network and hence respects the power supply limit

- Price Coordinator - The price coordinator detailed in Section 2.3 is used to attempt to respect the power supply limit

The price coordinator's performance depends on a number of parameters, principally the value of $u^{\max }$ and the relative ratios of $Q / R$ and $Q / r$, and the effect of these is investigated in Section 4.1.4. This Section however aims to give an initial insight into how the price coordinator behaves and performs, and hence the values chosen for these parameters are arbitrary to some extent. To this end, the power profiles for the building network in the 3 cases outlined above are shown in Fig. 4 with performance metrics summarised in Table 5. Fig. 5 presents iterations of the price coordinator for insight into how it 'negotiates' with the building network. Simulation parameters are listed in Table 6, which were kept the same for all 3 cases.
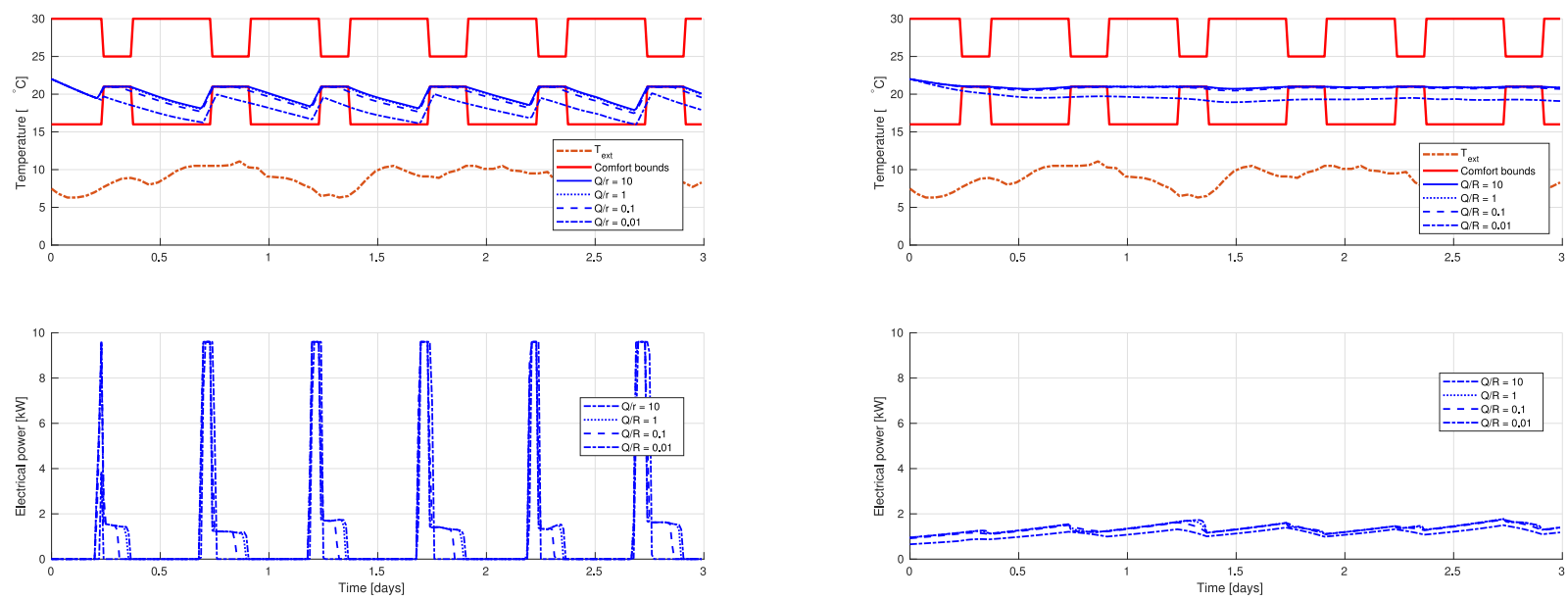

(a) Linearly penalised system inputs

(b) Quadratically penalised system inputs

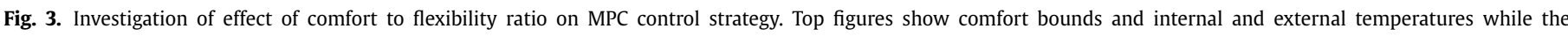
bottom figures show the electrical power drawn by the heat pumps. 

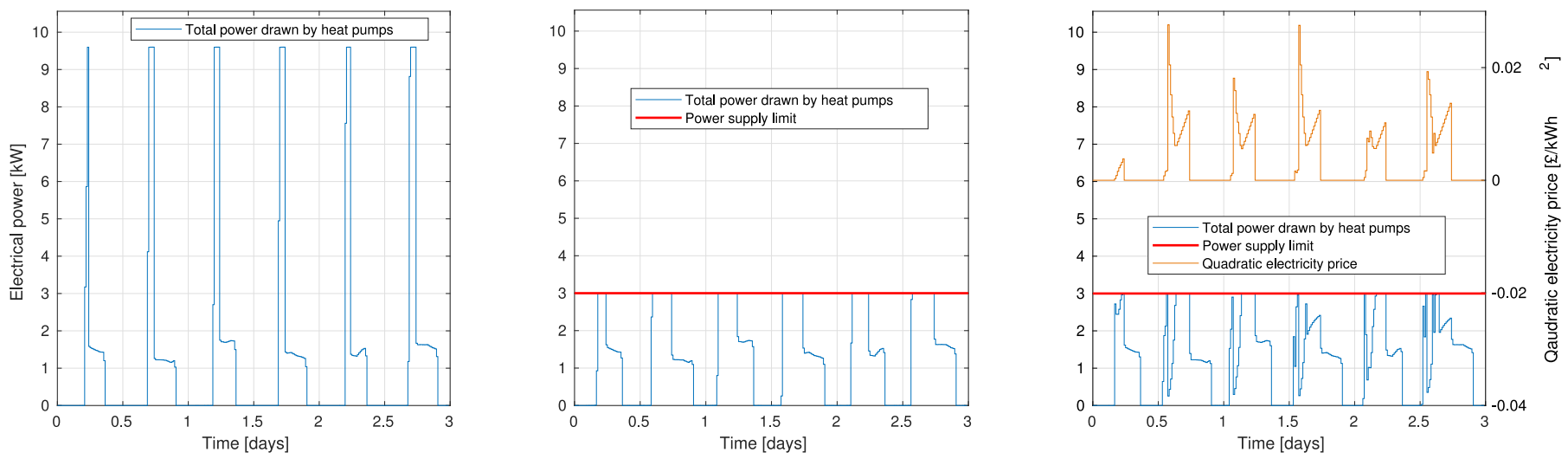

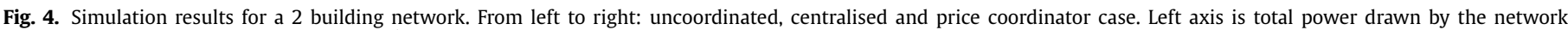
while right axis is the electricity price $p_{k}^{\text {quad }}$ resulting from the price coordinator simulation.

Table 6

2 building network simulation parameters. Note that the value of $u^{\max }$ is not applicable in the uncoordinated case.

\begin{tabular}{ll}
\hline Parameter [Units] & Value \\
\hline$u^{\max }[\mathrm{kW}]$ & 3 \\
$Q\left[£ / \mathrm{siK}^{2}\right]$ & 10 \\
$R[1 / \mathrm{kWh}]$ & 1 \\
$r[-]$ & 1 \\
Simulation time [days] & 3 \\
$N[\#$ of time steps] & 96 \\
{$\left[p^{\text {lin }}, p^{\text {quad }}\right][£ / \mathrm{kWh}]$} & {$[0.1,0]$} \\
Initial temperature $\left[{ }^{\circ} \mathrm{C}\right]$ & 22 \\
\hline
\end{tabular}

Table 7

Simulation parameters for Pareto analysis. 30 logarithmically spaced points were used for $R$ (and therefore $Q / R$ ) while 3 different values of $u^{\max }$ were used.

\begin{tabular}{|c|c|c|c|}
\hline Parameter [Units] & & Value & \\
\hline$u^{\max }[\mathrm{kW}]$ & 3 & 1.5 & 1 \\
\hline$Q\left[f / \operatorname{siK}^{2}\right]$ & & 10 & \\
\hline$R[1 / \mathrm{kWh}]$ & & $1-1000$ & \\
\hline$r[-]$ & & 1 & \\
\hline Simulation time [days] & & 3 & \\
\hline$N$ [\# of time steps] & & 96 & \\
\hline$\left[p^{\text {lin }}, p^{\text {quad }}\right][£ / \mathrm{kWh}]$ & & {$[0.1,0]$} & \\
\hline Initial temperature $\left[{ }^{\circ} \mathrm{C}\right]$ & & 22 & \\
\hline
\end{tabular}

Fig. 4 shows how the CMPC and price coordinator are both capable of reducing the peak power demand and hence respect the power supply limit. By comparing these with the uncoordinated case and observing the CBV values in Table 5, it is clear that both coordination methods reduce this peak by spreading the power demand over a longer period of time without sacrificing user comfort. The price coordinator's alterations of the quadratic electricity price causes erratic load profiles compared to the centralised case however, though surprisingly for this case this does not lead to an increase in total energy consumption or comfort violations, though the power supply limit violations do increase.

Fig. 5 shows how the price coordinator 'negotiates' with the building network in the first time step $k=0$. Despite the high value of $Q / R$, the small change in $p_{i \mid j, k}^{\text {quad }}$ at $j=1$ is able to influence the load profile of the network at the next price iteration $j=2$. As these iterations progress, the peak occurring roughly at $i=20$ is reduced to the point that it almost satisfies the power supply limit. This is not the case for the peak that occurs at $i \approx 60$, which the price coordinator tries to reduce by increasing the price ever more. This is ineffective however, since the optimal solution of the MPCs becomes to draw more power where $p_{i \mid j, k}^{q u a d}=0$ and so the peak is only displaced. This explains the erratic load profile seen in the price coordinator case of Fig. 4. For the timestep shown in Fig. $5, j^{\max }$ is reached without satisfying the power supply limit constraint, and so $u_{b, i=0 \mid j=j^{\max }, k=0}^{*}$ is applied to all $b \in \mathcal{B}, k$ is set to $=1$ and the price coordinator coordination process is repeated starting at $j=1$.

\subsubsection{Pareto analysis of price coordinator performance}

This Section analyses the effect of the comfort to flexibility ratio $Q / R$ on the performance of the price coordinator. To do this, Pareto curves for various values of $u^{\max }$ were produced as shown in Fig. 6, plotting $E_{\text {total }}$ against $\mathrm{CBV}$ and displaying maximum power supply limit violations (a measure of peak reduction) using a colour gradient. $E_{\text {total }}$ was used as a performance metric since for constant $p_{k}^{\text {lin }}$ this is the same as the total electricity costs, while being less ambiguous given the use in this paper of quadratic electricity price. Numbers above data points indicate the value of $Q / R$ to show the direction of increasing $Q / R$ on the Pareto curve, while the result for a CMPC, which only has a linear price penalty, is shown for comparison. Since the total electrical power drawn by the heat pumps in the 2 building network during occupancy was approximately 1.5 $\mathrm{kW}$ (see Fig. 3a), values of the power supply limit above, below and at this value were chosen for this analysis. The parameters used for this analysis are shown in Table 7.

Fig. 6 shows the effect of the tuning parameter $Q / R$ on the trade off between comfort and total cost. In Fig. 6a, the high value of $u^{\max }=3$ means that reducing the network's peak load does not require a significant trade off - the building network is able to reduce its peak power consumption without sacrificing total energy cost or comfort violations, as indicated by the CMPC solution which is located in the bottom left of Fig. 6. Here, a high value of $Q / R$ is advantageous as significant peak reduction is not needed. The high value of $Q / R$ softens the impact of the quadratic energy component in the objective, thus favouring energy minimisation over peak reduction, i.e. the term $r p_{i \mid k}^{l i n} u_{b, i \mid j, k}$ still dominates over $R p_{i \mid j, k}^{q u a d} u_{b, i \mid j, k}^{2}$ in the MPC objective function. Conversely, as $Q / R$ reduces, so to does the emphasis on energy minimisation and as such, energy consomption increases. Below a $Q / R$ ratio of approximately 0.1 , the quadratic energy component begins to dominate the comfort component of the cost, leading to a reduction in energy consumption at the expense of an increase in comfort violation. In Fig. 6b, a more traditional Pareto curve is observed in which comfort comes at the cost of an increase in energy consumption though there is still an initial trade off between peak reduction and energy minimisation for high $Q / R$. Fig. 6c represents a case where comfort boundaries have to be violated in order to satisfy the power 

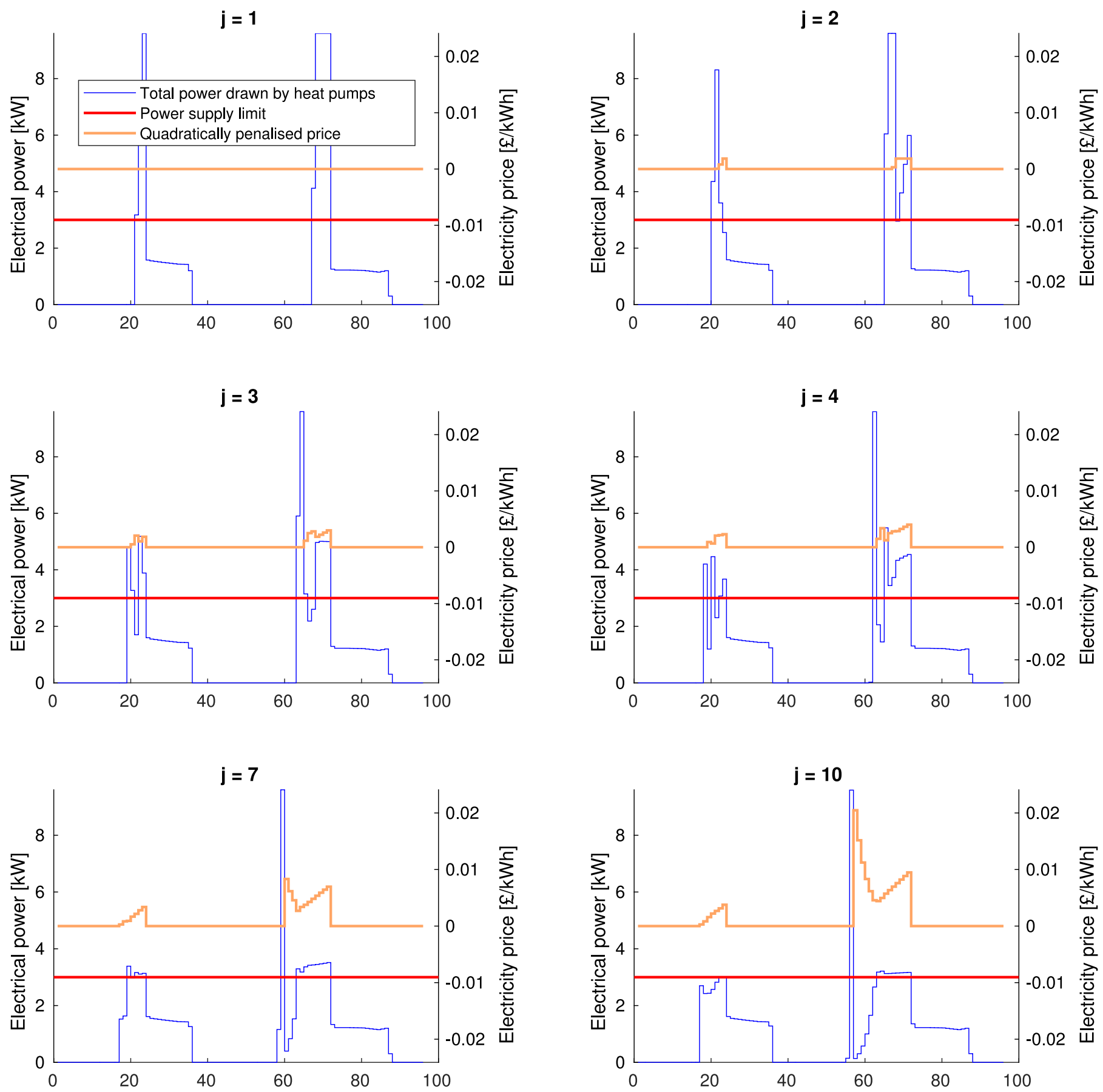

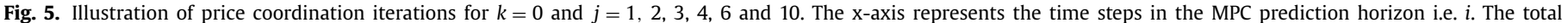
power drawn by the heat pumps is then $u_{i \mid j, k}^{\text {sum }}$ plotted over $i$, and similarly for $p_{i \mid j, k}^{\text {quad }}$.

supply limit. To achieve this, the quadratic energy component must dominate the comfort violation component of the objective and as such, a low value for the $Q / R$ ratio is required to ensure the price coordinator impact to be sufficient. This is emphasised by the high level of comfort violation produced by the CMPC. Note that while the CMPC requires complete knowledge of the entire network, the price-coordinator does not. To achieve a competitive performance however, the price-coordinator must know the level of flexibility (captured here as the $Q / R$ ratio) suited to the system.

It is clear from this that the required tuning of the ratio $Q / R$ is dependent on the $u^{\max }$ (and of course on the behaviour of the price controller) - a low value of $u^{\max }$ which requires comfort bounds to be violated requires a low value of $Q / R$ (see Fig. 6c) for the electricity price increases chosen here. When comfort bounds do not have to be violated in order to satisfy the power supply limit, the value of $Q / R$ matters less ((see Fig. 6a and 6b), though lower values may lead to unnecessary trade offs between total energy consumption and thermal comfort. This disadvantages the price coordinator compared to the centralised controller in that the required flexibility of the system (i.e. the tuning of $Q / R$ ratio) is not necessarily known in advance.

\subsection{0 building network case study}

In an analagous way to Section 4.1.3, the 100 building network described in Section 3.2 was simulated for 3 different cases, 


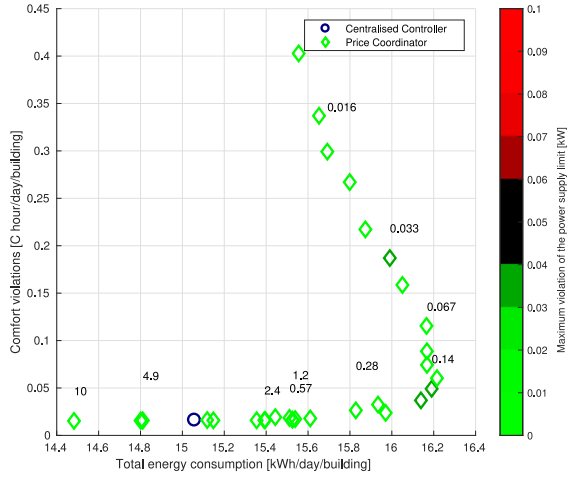

(a) $u^{\max }=3$

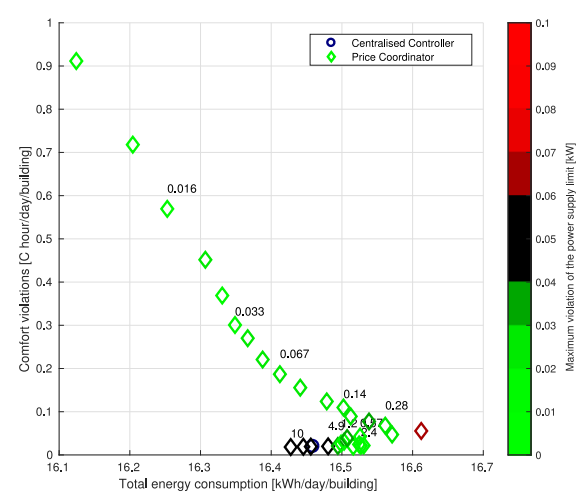

(b) $u^{\max }=1.5$

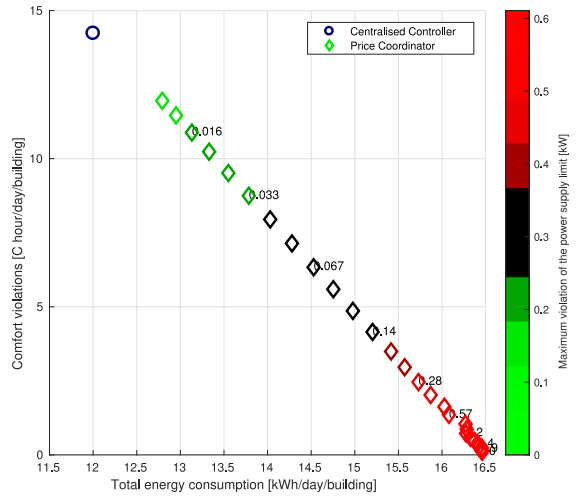

(c) $u^{\max }=1$

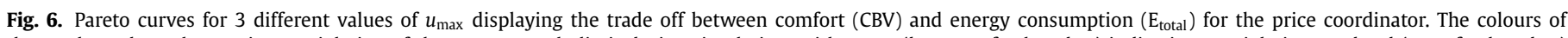

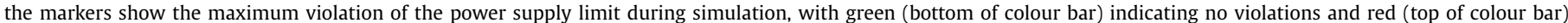

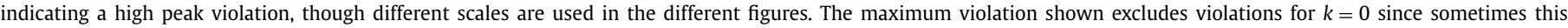

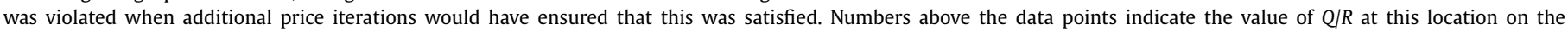

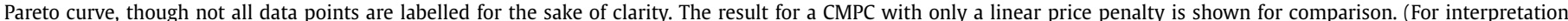
of the references to colour in this figure legend, the reader is referred to the web version of this article.)
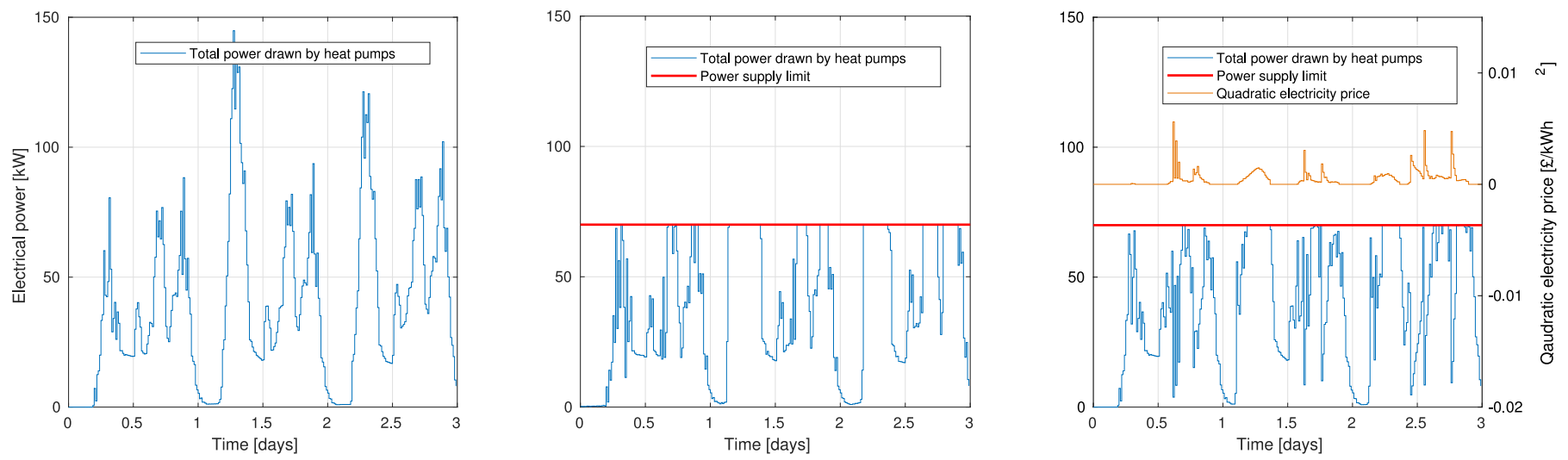

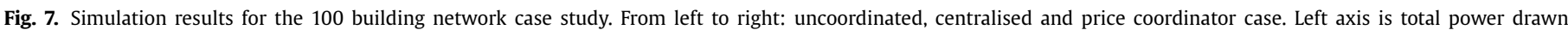
by the network while right axis is the electricity price $p_{k}^{\text {quad }}$ resulting from the price coordinator simulation.

Table 8

Simulation parameters for the 100 building network case study. Note that the value of $u^{\max }$ is not applicable to the uncoordinated case.

\begin{tabular}{ll}
\hline Parameter [Units] & Value \\
\hline Simulation time [days] & 3 \\
$R[1 / \mathrm{kWh}]$ & 10 \\
Horizon length [\# of samples] & 96 \\
$r[-]$ & 1 \\
{$\left[p^{\text {lin }}, p^{\text {quad }}\right][£ / \mathrm{kWh}]$} & {$[0.1,0]$} \\
$Q\left[£ / \mathrm{siK}^{2}\right]$ & 10 \\
Initial temperature $\left({ }^{\circ} \mathrm{C}\right)$ & 22 \\
$u^{\max }(\mathrm{kW})$ & 70 \\
\hline
\end{tabular}

uncoordinated, centralised and price coordinator. The power supply limit was chosen to be $70 \mathrm{~kW}$, since this intersected many of the peaks in power consumption while not being so constraining that the price controller would have to violate comfort bounds. Fig. 7 displays the heat pump total power profiles $u_{k}^{\text {sum }}$ for the 3 cases while Table 9 compares their performance metrics. The parameters used for the 100 building network simulation are shown in Table 8 and were the same for all cases.

From Fig. 7, it is evident that both the centralised controller and the price coordinator are able to satisfy the power supply
Table 9

Performance metrics for simulations of 100 building network for 3 different cases. Simulation times were recorded on an HP Elitebook 840 G2 with Intel Core i55200U CPU 2.20GHz 4, 4 GiB of RAM running on 64-bit Ubuntu.

\begin{tabular}{llll}
\hline Performance Metric & Uncoordinated & Centralised & Price Coordinator \\
\hline $\mathrm{E}_{\text {total }}$ & 9.1921 & 9.2459 & 9.3230 \\
CBV & 0.0114 & 0.0070 & 0.0110 \\
PSLV & 0.8971 & 0 & 0.00028794 \\
Simulation Time [min] & 18 & 135 & 39 \\
\hline
\end{tabular}

limit, and both achieve this by spreading the peaks in $u_{k}^{\text {sum }}$ over a longer period of time and therefore reducing their size. As noted in Section 4.1.3, the building network's load profile is more erratic for the price coordinator's case compared to the centralised case, though the increased building network size dampens this effect to some degree. Table 9 shows that the centralised approach is better than the price coordinator in terms of energy usage $(0.7 \%)$, comfort boundary violations and power supply limit violations, though negligibly so - the yearly increase in energy usage would amount to a difference of merely $7 \mathrm{kWh} /$ building assuming 90 similar winter days. The centralised controller however took almost 8 times longer to simulate compared to the uncoordinated case, while the price coordinator only twice as long. 


\subsection{Discussion of results and suggestions for future work}

The results of Section 4.2 show that the price coordinator is able to achieve its objective of satisfying the power supply limit constraint while comparing favourably with the centralised case, with similar key performance metrics and a fourfold decrease in computation time. This approach is highly flexible since the objective function of the MPCs contains three terms minimising energy consumption or costs, comfort violations and peak magnitudes, allowing for an a priori trade off to between these three objectives to be explored. The price coordinator algorithm used here is however quite crude, since some increases in $p_{i \mid j, k}^{\text {quad }}$ have no effect on the MPCs control strategy while others are unnecessarily large (see Fig. 5). More sophisticated methods for choosing $p_{j, i \mid k}^{q u a d}$ at each iteration, based on machine learning for example, could decrease the number of iterations and hence computation time while leading to solutions closer to the centralised case. Future work could also look at incorporating a time varying tariff for $p_{k}^{l i n}$ as is the case with some retailers (e.g. Octopus energy in the UK) to see what effect, if any, this has on the price coordinator's performance.

This approach nonetheless suffers from a number of disadvantages. While the multi-objective formulation of the MPC is flexible, Section 4.1.4 demonstrated that low tunings of $Q / R$ could lead to unnecessary trade offs, e.g. sacrificing total energy and comfort violations to satisfy the power supply limit when the centralised case shows that this is possible without any significant trade off. Competitive performance of the price controller therefore requires tuning which may not be possible to do adequately a priori. Further work on the price coordinator could address this point by developing an algorithm that tuned the price coordinator and MPC parameters online. The price coordinator approach is also not well suited for other coordination problems, such as building zones with interacting dynamics, without modification to the price coordinator. Finally, if the goal is to satisfy the power supply limit at all costs, there is no guarantee of doing this for a finite number of price iterations and the resulting solution is not guaranteed to be close to that of the centralised case.

\section{Conclusion}

In this paper, a hierarchical price coordinator was used to coordinate heat pumps in a building network in the presence of a power supply limit. The price coordinator method is a satisfyingly intuitive way of achieving coordination since peaks in power demand are reduced by increasing a pseudo electricity price, though a quadratic electricity price was used since a linear electricity price was not suitable for coordination. This approach is advantageous since it requires little information exchange between the MPCs and the building network while scaling better with the network size and being more reliable than the centralised case. It is also flexible, since a trade off between minimising total costs, comfort violations and peak magnitudes can be done explicitly. In its current form however, the proposed method could lead to unnecessary trade offs between these 3 criteria, warranting future research into algorithms for the price coordinator that improve on the proportional based method presented here. Finally, a 100 building network case study was presented which demonstrated that the price coordinator was able to perform similarly to the centralised case, with an increase in energy consumption of less than $7 \mathrm{kWh}$ per building per year.

\section{Declaration of competing interest}

None.

\section{Supplementary material}

Supplementary material associated with this article can be found, in the online version, at doi:10.1016/j.enbuild.2019.109421.

\section{References}

[1] L. Prez-Lombard, J. Ortiz, C. Pout, A review on buildings energy consumption information, Energy Build. 40 (3) (2008) 394-398, doi:10.1016/j.enbuild.2007. 03.007.

[2] P.H. Shaikh, N.B.M. Nor, P. Nallagownden, I. Elamvazuthi, T. Ibrahim, A review on optimized control systems for building energy and comfort management of smart sustainable buildings, Renewable Sustainable Energy Rev. 34 (Supplement C) (2014) 409-429, doi:10.1016/j.rser.2014.03.027.

[3] EPBD, Directive 2010/31/EU of the European Parliament and of the Council of 19 May 2010 on the energy performance of buildings (recast), Off. J. Eur. Union L153 (2010) 13-35.

[4] D. Connolly, H. Lund, B. Mathiesen, S. Werner, B. Mller, U. Persson, T. Boermans, D. Trier, P. stergaard, S. Nielsen, Heat roadmap europe: combining district heating with heat savings to decarbonise the eu energy system, Energy Policy 65 (2014) 475-489, doi:10.1016/j.enpol.2013.10.035.

[5] E. O’Dwyer, I. Pan, S. Acha, N. Shah, Smart energy systems for sustainable smart cities: Current developments, trends and future directions, Appl. Energ. 237 (2019) 581-597.

[6] M. Maasoumy, A. Sangiovanni-Vincentelli, Smart connected buildings design automation: foundations and trends, Found. Trends Electron. Des.Autom. 10 (1-2) (2016) 1-143, doi:10.1561/1000000043.

[7] A. Afram, F. Janabi-Sharifi, Theory and applications of HVAC control systems a review of model predictive control (MPC), Build. Environ. 72 (2014) 343-355, doi:10.1016/j.buildenv.2013.11.016.

[8] Y. Zong, G.M. Bning, R.M. Santos, S. You, J. Hu, X. Han, Challenges of implementing economic model predictive control strategy for buildings interacting with smart energy systems, Appl. Therm. Eng. 114 (2017) 1476-1486, doi:10.1016/j.applthermaleng.2016.11.141.

[9] C.R. Touretzky, M. Baldea, Integrating scheduling and control for economic MPC of buildings with energy storage, J. Process Control 24 (8) (2014) 12921300. Economic nonlinear model predictive control. doi: $10.1016 / \mathrm{j}$.jprocont. 2014.04.015.

[10] D.I. Mendoza-Serrano, D.J. Chmielewski, HVAC control using infinite-horizon economic MPC, Proc. IEEE Conf. Decis.Control 60616 (3) (2012) 6963-6968, doi:10.1109/CDC.2012.6426071.

[11] P.-D. Morosan, R. Bourdais, D. Dumur, J. Buisson, Building temperature regulation using a distributed model predictive control, Energy Build. 42 (9) (2010) 1445-1452, doi:10.1016/j.enbuild.2010.03.014.

[12] C. Wu, W. Gu, P. Jiang, Z. Li, H. Cai, B. Li, Combined economic dispatch considering the time-delay of district heating network and multi-regional indoor temperature control, IEEE Trans. Sustainable Energy 9 (1) (2018) 118-127, doi:10.1109/TSTE.2017.2718031.

[13] P. Stadler, A. Ashouri, F. Marechal, Distributed model predictive control of energy systems in microgrids, in: 10th Annual International Systems Conference, SysCon 2016 - Proceedings, 2016, pp. 1-6, doi:10.1109/SYSCON.2016.7490607.

[14] A. Staino, H. Nagpal, B. Basu, Cooperative optimization of building energy systems in an economic model predictive control framework, Energy Build. 128 (2016) 713-722, doi:10.1016/j.enbuild.2016.07.009.

[15] E. McKenna, I. Richardson, M. Thomson, Smart meter data: balancing consumer privacy concerns with legitimate applications, Energy Policy 41 (2012) 807814, doi:10.1016/j.enpol.2011.11.049.

[16] H. Scherer, M. Pasamontes, J. Guzmn, J. Ivarez, E. Camponogara, J. Normey-Rico, Efficient building energy management using distributed model predictive control, J. Process Control 24 (6) (2014) 740-749. Energy Efficient Buildings Special Issue. doi: 10.1016/j.jprocont.2013.09.024.

[17] M.Y. Lamoudi, M. Alamir, P. Beguery, Distributed constrained model predictive control based on bundle method for building energy management, in: IEEE Conference on Decision and Control and European Control Conference, IEEE, 2011, pp. 8118-8124, doi:10.1109/cdc.2011.6161076.

[18] J.B. Rawlings, N.R. Patel, M.J. Risbeck, C.T. Maravelias, M.J. Wenzel, R.D. Turney, Economic MPC and real-time decision making with application to large-scale HVAC energy systems, Comput. Chem. Eng. 114 (2018) 89-98, doi:10.1016/j. compchemeng.2017.10.038.

[19] A. Parisio, C. Wiezorek, T. Kyntj, J. Elo, K. Strunz, K.H. Johansson, Cooperative MPC-based energy management for networked microgrids, IEEE Trans. Smart Grid 8 (6) (2017) 3066-3074, doi:10.1109/TSG.2017.2726941.

[20] N. Li, L. Chen, S.H. Low, Optimal demand response based on utility maximization in power networks, IEEE Power and Energy Society General Meeting, 2011, doi:10.1109/PES.2011.6039082.

[21] M. Diekerhof, S. Schwarz, A. Monti, Distributed optimization for electrothermal heating units, in: 2016 IEEE PES Innovative Smart Grid Technologies Conference Europe (ISGT-Europe), 2016, pp. 1-6, doi:10.1109/ISGTEurope.2016. 7856258.

[22] G. Strbac, D.S. Kirschen, Fundamentals of Power System Economics, John Wiley \& Sons, Incorporated, Newark, 2018, pp. 125-131.

[23] J. Chimento, S. Gonzato, E. O'Dwyer, G.B.-T. an Salvador Acha, N. Shah, District-level coordination of predictive control strategies for urban 
residential heating networks, 2018. https://www.researchgate.net/publication/ 327542662_District-level_coordination_of_predictive_control_strategies_for_ urban_residential_heating_networks.

[24] F. Oldewurtel, A. Parisio, C.N. Jones, D. Gyalistras, M. Gwerder, V. Stauch, B. Lehmann, M. Morari, Use of model predictive control and weather forecasts for energy efficient building climate control, Energy Build. 45 (2012) 15-27, doi:10.1016/j.enbuild.2011.09.022.

[25] J. Chimento, S. Gonzato, District-level Coordination of Predictive Control Strategies for Urban Residential Heating Networks, Imperial College London, Prince Consort Road, 2017 Master's thesis.
[26] G. Bustos-Turu, Integrated Modelling Framework for the Analysis of Demand Side Management Strategies in Urban Energy Systems, Imperial College London, Imperial College London, 2018 Ph.D. thesis.

[27] D. Fischer, H. Madani, On heat pumps in smart grids: a review, Renewable Sustainable Energy Rev. 70 (C) (2017) 342-357, doi:10.1016/j.rser.2016.12.01. 